This is a pre-print of the paper published in Acta Geotechnica, 2021,16(1):77-91. https://doi.org/10.1007/s11440-020-01076-7

The mechanical behaviors of methane hydrate-bearing sands (MHBS) are largely affected by the presence of methane hydrate, temperature, and pore pressure. In this study, we present a simple hypoplastic model for MHBS. Methane hydrate saturation is included as a state parameter affecting the mechanical behaviors of MHBS. A new phase parameter is introduced to account for the coupled effects of temperature and pore pressure on the mechanical behaviors of MHBS. The phase parameter can be determined by a simple function of temperature and pore pressure. Comparison of the predictions with experiments shows that the model is able to capture the salient behaviors of MHBS.

\section{A state-dependent hypoplastic model for methane hydrate-bearing sands} University, Shanghai, China, 200092

Vienna, Austria

Keywords: Hypoplastic constitutive model, Methane hydrate, Sands, Phase state, Critical state

\section{Introduction}

Methane hydrate $(\mathrm{MH})$ is a crystalline clathrate composed of methane and water molecules. It is widely distributed beneath permafrost and in marine continental margin sediments. $\mathrm{MH}$ can be used to extract methane gas, and has attracted global interests as a promising energy resource [1]. According to field investigations, MHs primarily exist in the forms of discrete nodules or veins in fine-grained sediments or disseminate in the pore spaces of coarse sands. Compared to fine-grained sediments, sand reservoirs are more

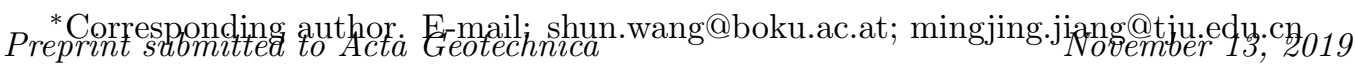


attractive for $\mathrm{MH}$ exploitation due to the high-permeability of sand media and the high-concentration of $\mathrm{MHs}[2,3]$. However, the exploitation of $\mathrm{MH}$ may destabilize methane hydrate-bearing sands (MHBS) and potentially lead to the unsteady deformation of seabed [4], in-stability of production well [5], and even marine landslides [6]. It is therefore crucial to properly characterize the mechanical behaviors of MHBS for MH exploitation.

The mechanical behaviors of MHBS have been extensively investigated by laboratory tests. Sufficient experimental results [7-13] show that the stiffness, strength, and dilation of MHBS are enhanced by increasing MH saturation, which is defined as the ratio of $\mathrm{MH}$ volume to sediment pore volume. Besides, the mechanical behaviors of MHBS are markedly influenced by pore pressure and temperature $[7,13]$. For example, the peak strength of MHBS increases by $18 \%$ when temperature decreases from $10^{\circ} \mathrm{C}$ to $1{ }^{\circ} \mathrm{C}$ [7]; and a $40 \%$ increase of the peak strength is observed when pore pressure increases from $5 \mathrm{MPa}$ to $10 \mathrm{MPa}$ [13]. Hence, the $\mathrm{MH}$ saturation, temperature, and pore pressure dependences are important in modeling the mechanical behaviors of MHBS.

During the last decade, a great effort has been made in the development of constitutive models for predicting the mechanical behaviors of MHBS. Earlier models are usually developed based on the Ducan-Change (DC) theory [14-16] and the Mohr-Coulomb (MC) theory [17-20]. In these constitutive models, MH saturation and temperature are considered as important factors that influence the mechanical behaviors of MHBS. The DC type models establish a stress-strain relationship through a hyperbolic function, in which the initial stiffness and the peak shear strength can be related to confining pressure [14], MH saturation [15], and temperature [14, 16]. With a nonlinear elastic formulation, the nonlinear behavior of MHBS can be described, while the applied hyperbolic function is usually too simple to fully capture the volumetric and strain softening behaviors. In the MC type models, the effect of $\mathrm{MH}$ saturation can be considered by including the dependency of cohesion on MH saturation [17-20]. However, without a yielding surface, the MC type models predict inaccurate volumetric deformation of MHBS under loading.

Although the aforementioned models are able to describe some basic features of MHBS, the prediction of the critical state behavior, an important aspect of the behavior of MHBS, is beyond the capacity of the models due to the limitation of model framework. In contrast to these models, the critical state models based on the Modified Cam-Clay framework consider the effects of $\mathrm{MH}$ saturation using the critical state parameters. The volumetric change, 
strain softening, and MH-induced bonding can be reasonable described [21$25]$. Thus, the critical state models are among the most common options in predicting the mechanical behavior of MHBS.

A closer inspection of the relevant literature reveals, however, that the effects of temperature and pore pressure, have rarely been included in available critical state models. Although an attempt has been made to account for the effects of temperature and pore pressure within the framework of bounding surface plasticity [23], the formulation of the constitutive equation is complicated, which may hinder the understanding and application of the model. From the practical point of view, a constitutive model with simple formulation while is capable of capturing the salient behaviors of MHBS is to be favoured.

Hypoplastic models describe the behaviors of granular materials by nonlinear tensorial functions [26]. Unlike elastoplastic models, hypoplastic models need not to predefine yield surface, plastic potential, hardening rule, flow rule, and the decomposition of deformation into elastic and plastic parts [26]. Owing to their simple formulations with few model parameters, hypoplastic models have been widely used to model the behaviors of sands [27-30], clay [31, 32], frozen soil [33], and debris materials [34], etc. Recently, the hypoplstic model has also been applied to model the mechanical behaviors of MHBS [35]. The effects of MH saturation and confining pressure on the stress-strain and volumetric responses can be well captured. However, the parameters for different MH saturations and confining pressures are not unified, i.e., need to be recalibrated. Moreover, some salient factors, such as void ratio, temperature, and pore pressure, are not discussed.

This study aims to develop a hypoplastic model accounting for the effects of MH saturation, stress level, void ratio, temperature, and pore pressure. First, a simple hypoplastic model for sand [36] is enhanced by incorporating the critical state concept. Second, the effect of $\mathrm{MH}$ is incorporated by considering both the hydrate-induced bonding and the critical state line (CSL) being dependent on $\mathrm{MH}$ saturation, temperature, and pore pressure. In particular, a phase parameter is introduced to account for the coupled effects of temperature and pore pressure on the mechanical behaviors of MHBS. After that, the calibration of the model is illustrated. Last, the model is validated by comparing simulation results with experiments. The performance of the model and some limitations are also discussed. 


\section{Hypoplastic model with critical state for sand}

Wu et al. [36] proposed a basic constitutive model for sand. This model contains tensor polynomial with three linear terms and one nonlinear term in strain rate:

$$
\stackrel{\circ}{\boldsymbol{\sigma}}=C_{1}(\operatorname{tr} \boldsymbol{\sigma}) \dot{\boldsymbol{\epsilon}}+C_{2}(\operatorname{tr} \boldsymbol{\sigma})(\operatorname{tr} \dot{\boldsymbol{\epsilon}}) \mathbf{I}+C_{3} \frac{\operatorname{tr}(\boldsymbol{\sigma} \dot{\boldsymbol{\epsilon}})}{\operatorname{tr} \boldsymbol{\sigma}} \boldsymbol{\sigma}+C_{4}\left(\boldsymbol{\sigma}+\boldsymbol{\sigma}^{*}\right)\|\dot{\boldsymbol{\epsilon}}\|
$$

where $C_{i},(i=1,2,3,4)$ are dimensionless parameters, the sign tr denotes the trace of a tensor, $\boldsymbol{\sigma}$ is the Cauchy stress tensor, $\dot{\boldsymbol{\epsilon}}$ is the strain rate (stretching) tensor, $\mathbf{I}$ is the second-order unit tensor, $\boldsymbol{\sigma}^{*}$ is the deviatoric stress tensor defined as $\boldsymbol{\sigma}^{*}=\boldsymbol{\sigma}-(\operatorname{tr} \boldsymbol{\sigma}) \mathbf{I} / 3,\|\dot{\boldsymbol{\epsilon}}\|$ is the Euclidean norm of the strain rate, and $\stackrel{\boldsymbol{\sigma}}{ }$ is the Jaumann stress rate defined as:

$$
\stackrel{\circ}{\sigma}=\dot{\sigma}+\sigma \dot{\omega}+\dot{\omega} \sigma
$$

where $\dot{\boldsymbol{\sigma}}$ is the material time derivative of the Cauchy stress, and $\dot{\boldsymbol{\omega}}$ is the spin tensor.

This basic model takes the stress tensor as the only state variable. As a consequence, different parameters are required for the same material but with different initial densities. This shortcoming, however, can be readily remedied by integrating the critical state concept. To this end, a density function $f_{\mathrm{d}}$ [37] is introduced to account for the effect of void ratio. The basic model (1) is then recast as follows:

$$
\stackrel{\sigma}{\boldsymbol{\sigma}}=C_{1}(\operatorname{tr} \boldsymbol{\sigma}) \dot{\boldsymbol{\epsilon}}+\left(1-f_{\mathrm{d}}\right) C_{2}(\operatorname{tr} \boldsymbol{\sigma})(\operatorname{tr} \dot{\boldsymbol{\epsilon}}) \mathbf{I}+C_{3} \frac{\operatorname{tr}(\boldsymbol{\sigma} \dot{\boldsymbol{\epsilon}})}{\operatorname{tr} \boldsymbol{\sigma}} \boldsymbol{\sigma}+f_{\mathrm{d}} C_{4}\left(\boldsymbol{\sigma}+\boldsymbol{\sigma}^{*}\right)\|\dot{\boldsymbol{\epsilon}}\|
$$

With the density function $f_{\mathrm{d}}$, this model is able to consider variation of the density during shearing. Several forms of density functions can be found in the literature. In this work, a simple form suggested by Wang et al. [38] is used. It reads:

$$
f_{\mathrm{d}}=\left(\frac{e}{e_{\mathrm{crt}}}\right)^{\alpha}
$$

where $e$ is the current void ratio, $e_{\text {crt }}$ is the critical state void ratio, and $\alpha$ is a constitutive constant that controls the degree of strain softening [29, 38]. The value of $f_{\mathrm{d}}$ is less than 1 for a dense state, greater than 1 for a loose state, and equals 1 at the critical state. The critical state void ratio is determined 
by:

$$
e_{\mathrm{crt}}=e_{\Gamma}-\lambda \ln \left(\frac{p^{\prime}}{p_{\mathrm{a}}}\right)
$$

where $\lambda$ is a parametric constants, $p^{\prime}=\operatorname{tr} \boldsymbol{\sigma} / 3$ is the effective mean stress, $p_{\text {a }}$ $=101.325 \mathrm{kPa}$ is the atmospheric pressure, and $e_{\Gamma}$ is the critical state void at the atmospheric pressure.

The constitutive equation (3), however, does not account for the influence of the initial void ratio on the initial stiffness, and usually gives rise to a very stiff stress-strain curve in triaxial test simulations. To modulate the shape of the stress-strain curve, the right side of Eq. (3) is multiplied by a stiffness function $f_{\mathrm{s}}$ proposed by $\mathrm{Wu}$ and Kolymbas [36]:

$$
f_{\mathrm{s}}=\frac{\exp \left[\beta\left(e_{\mathrm{crt}}-e\right)\right]}{(1+r)^{2}}
$$

where $\beta$ is a material parameter and $r$ is the stress ratio $\left\|\boldsymbol{\sigma}^{*}\right\| /(\operatorname{tr} \boldsymbol{\sigma})$. In this way, the constitutive model for the host sand is formulated as:

$$
\stackrel{\circ}{\boldsymbol{\sigma}}=f_{\mathrm{s}}\left[C_{1}(\operatorname{tr} \boldsymbol{\sigma}) \dot{\boldsymbol{\epsilon}}+\left(1-f_{\mathrm{d}}\right) C_{2}(\operatorname{tr} \boldsymbol{\sigma})(\operatorname{tr} \dot{\boldsymbol{\epsilon}}) \mathbf{I}+C_{3} \frac{\operatorname{tr}(\boldsymbol{\sigma} \dot{\boldsymbol{\epsilon}})}{\operatorname{tr} \boldsymbol{\sigma}} \boldsymbol{\sigma}+f_{\mathrm{d}} C_{4}\left(\boldsymbol{\sigma}+\boldsymbol{\sigma}^{*}\right)\|\dot{\boldsymbol{\epsilon}}\|\right]
$$

\section{Model extension to MHBS}

The mechanical behaviors of MHBS have been widely investigated by triaxial compression tests [7-13]. Experimental results reveal that the existence of $\mathrm{MH}$ enhances the strength and dilation of MHBS. A higher MH saturation usually leads to a larger cohesion and a higher location of the CSL on the $e-\log p^{\prime}$ plane. In addition, the strength and dilatancy of MHBS can be enhanced by a lower temperature and a higher initial pore pressure.

To account for the aforementioned effects, we proceed to improve the sand model (7) in two aspects. First, an internal variable $S$ is introduced to account for MH-induced cohesion. Second, a new phase parameter $L^{\prime}$ is proposed to describe the coupled effects of temperature and pore pressure on the cohesion and CSL of MHBS. The complete constitutive equations are given in 'Appendix' for clarity.

\subsection{Incorporation of cohesion}

Since the model (7) is homogeneous in stress, the limit surface in the principal stress space is a cone with its apex at the origin. As a result, Eq. 
(7) allows no tensile stress. This shortcoming can be removed by transforming the stress tensor $\boldsymbol{\sigma}$ in Eq. (7) by an internal variable $S$ :

$$
\boldsymbol{\sigma}_{\mathrm{T}}=\boldsymbol{\sigma}+S \mathbf{I}
$$

where $\boldsymbol{\sigma}_{\mathrm{T}}$ is a transformed stress tensor. In this way, the hydrate-induced cohesion can be included by replacing $\boldsymbol{\sigma}$ by $\boldsymbol{\sigma}_{\mathrm{T}}$ in Eq. (7).

As indicated by experimental results $[7,8]$, $\mathrm{MH}$ saturation $S_{\mathrm{MH}}$, temperature $T$, and pore pressure $P$ have significant influences on the $\mathrm{MH}$-induced cohesion. Therefore, the value of $S$ should be as a function of $S_{\mathrm{MH}}, T$, and $P$, i.e., $S=f\left(S_{\mathrm{MH}}, T, P\right)$. This function will be formulated in subsection 3.3.3 after introducing the new phase parameter $L^{\prime}$.

\subsection{The effect of $M H$ saturation}

Experimental results reveal that the critical state line of MHBS will shift upward on the $e-\log p^{\prime}$ plane with increasing $\mathrm{MH}$ saturation. Besides, the CSL of MHBS with a given $S_{\mathrm{MH}}$ is usually assumed to be parallel to that of the host sand [7], as shown in Fig. 1.

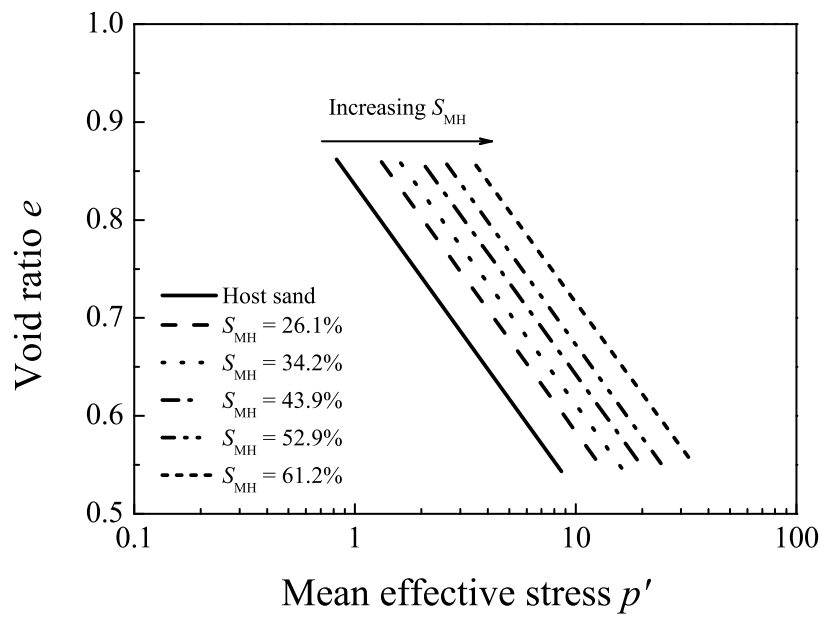

Figure 1: Critical state lines for MHBS [7].

Based on the above experimental observations, the effect of $\mathrm{MH}$ saturation on the CSL is suggested as:

$$
\begin{gathered}
e_{\mathrm{crt}}=e_{\Gamma(\mathrm{MH})}-\lambda \ln \left(p^{\prime} / p_{\mathrm{a}}\right) \\
e_{\Gamma(\mathrm{MH})}=e_{\Gamma}+\xi S_{\mathrm{MH}}^{\chi_{1}}
\end{gathered}
$$


where $\xi$ and $\chi_{1}$ are model parameters. The parameter $e_{\Gamma}$ is the same as that used in Eq. (5).

\subsection{The effects of temperature and pore pressure}

Previous experimental works show that the changes of temperature and pore pressure will lead to the variations of: (1) MH-induced cohesion and (2) the location of the CSL of MHBS [7, 8]. In the following, we proceed to consider the effects of temperature and pore pressure in a unified way by using a new phase parameter.

\subsubsection{A new phase parameter $L^{\prime}$}

The stability of MHs with respect to temperature and pore pressure can be described by a phase parameter $L$ defined by Hyodo et al. [39]. As illustrated in Fig. 2a, the phase parameter $L$ is defined as the minimum distance from a phase state $(\Omega)$ to the stability boundary. This boundary differentiates the area where MHs are stable (solid) or unstable (gaseous). Generally, MHs are stable only when the phase state lies above the stability boundary. Within this area, a higher value of $L$ indicates that the $\mathrm{MH}$ is more stable, and a higher strength can be gained [39], see Fig. 2b. Since the mechanical behaviors of MHBS are predominately governed by the properties of $\mathrm{MH}$, the effects of temperature and pore pressure on the mechanical behaviors of MHBS can be considered using the phase parameter $L$ [23, 40-43]. However, the value of $L$ can be obtained only by iterative method in previous studies due to the high nonlinearity of the stability boundary. To facilitate the use of the phase parameter concept, we will propose a simple stability boundary based on experimental results [44-50].

As shown in Fig. 3a, the stability boundary can be represented by two lines in the $T_{0} / T-\ln \left(P / P_{0}\right)$ plane. This characteristic suggests that the new phase parameter for a given phase state $(\Omega)$ can be expressed as a piecewise function as follows:

$$
L^{\prime}=\left\{\begin{array}{cll}
\frac{\left|35 \times\left(T_{0} / T\right)+\ln \left(P / P_{0}\right)-35\right|}{\sqrt{35^{2}+1^{2}}} & \Omega \in \text { area } & \mathrm{ABD} ; \\
\frac{\left|7.5 \times\left(T_{0} / T\right)+\ln \left(P / P_{0}\right)-7.5\right|}{\sqrt{7.5^{2}+1^{2}}} & \Omega \in \text { area } & \mathrm{CBD} ; \\
0 & \text { otherwise }
\end{array}\right.
$$




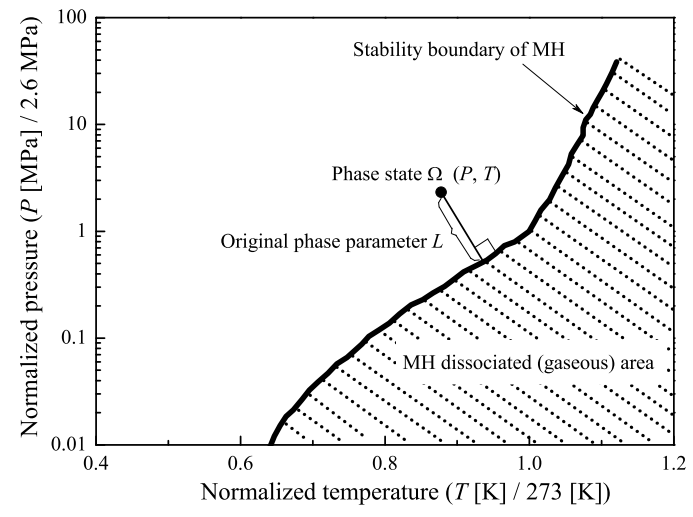

(a)

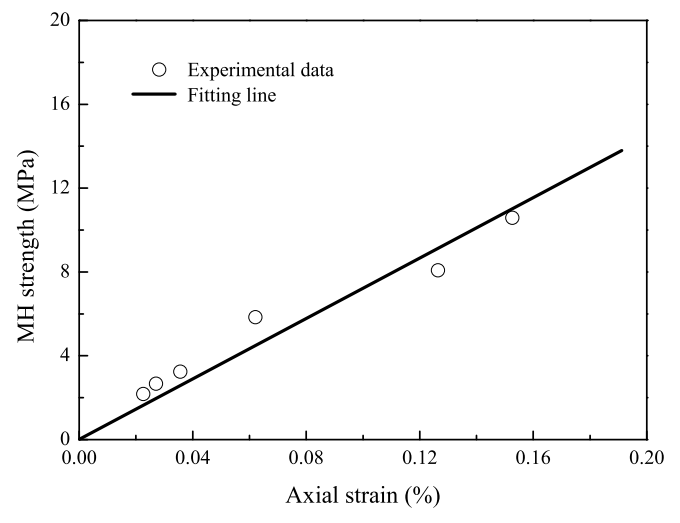

(b)

Figure 2: (a) Normalized temperature and pressure stability boundary and (b) relationship between the strength of MHBS and $L$ [39].

where $P$ and $T$ denote the current pore pressure and temperature, respectively; $P_{0}=2.23 \mathrm{MPa}$ and $T_{0}=273 \mathrm{~K}$ are the reference pore pressure and temperature, respectively; area $\mathrm{ABD}$ and $\mathrm{CBD}$ are the acute angle areas bounded by $\mathrm{ABD}$ and $\mathrm{CBD}$, respectively (see Fig. 3b, in which line $\mathrm{BD}$ is the angle bisector of $\mathrm{ABC}$ ). Compared to the phase parameter given in previous literature [23, 40-43], the new phase parameter $L^{\prime}$ is much simpler and can be explicitly determined.

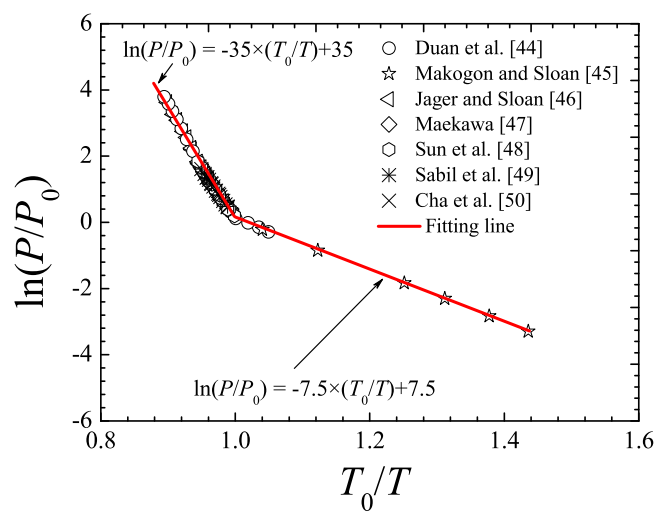

(a)

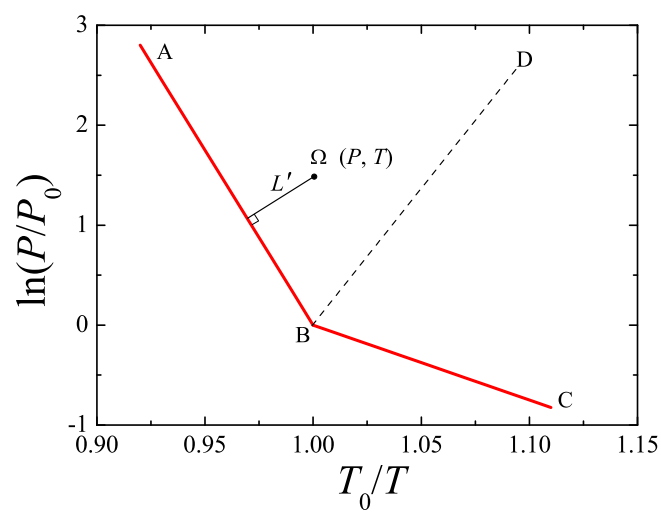

(b)

Figure 3: Stability boundary of methane hydrate in pure water: (a) experimental data and (b) the determining of the new phase parameter $L^{\prime}$. 


\subsubsection{The effect of $L^{\prime}$ on the CSL}

The relationship between the CSL of MHBS and the phase parameter $L^{\prime}$ can be established by assuming that the phase parameter changes only the position of the CSL rather than its slope (Fig. 4). The parameter controlling the position of the CSL of MHBS is considered to be dependent on the phase parameter $L^{\prime}$. The CSL of MHBS thus has the following formulation:

$$
\begin{gathered}
e_{\mathrm{crt}}=e_{\Gamma\left(L^{\prime}\right)}-\lambda \ln \left(p^{\prime} / p_{\mathrm{a}}\right) \\
e_{\Gamma\left(L^{\prime}\right)}=e_{\Gamma}+\xi L^{\prime \chi_{2}}
\end{gathered}
$$

where $\chi_{2}$ is an additional parameter controlling the effect of $L^{\prime}$ on the CSL of MHBS.

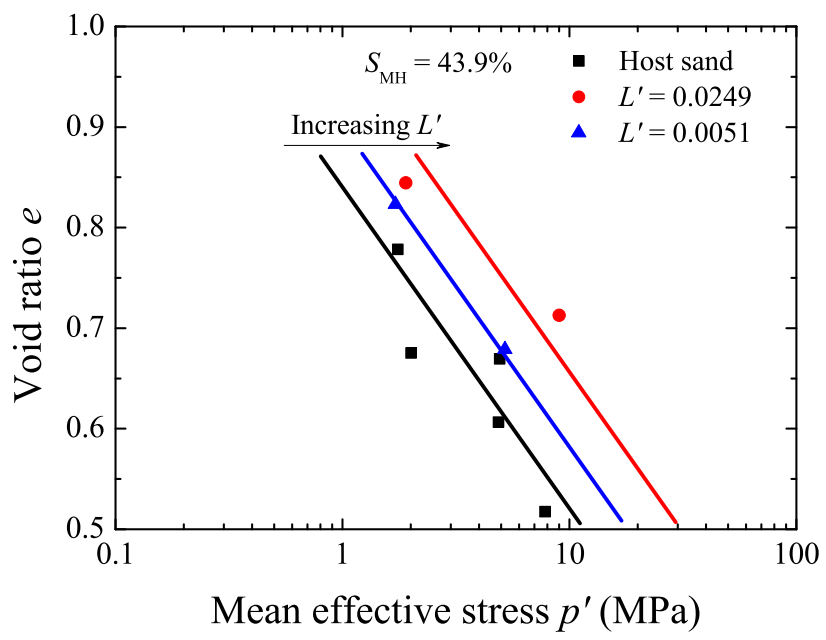

Figure 4: The influence of phase parameter $L^{\prime}$ on the CSL of MHBS (data from Hyodo et al. [7])

\subsubsection{The effects of $S_{M H}$ and $L^{\prime}$ on the CSL and cohesion}

Based on Eqs. (10) and (13), we proposed the following functions to account for the effects of $\mathrm{MH}$ saturation and phase state on the CSL of MHBS:

$$
\begin{gathered}
e_{\mathrm{crt}}=e_{\Gamma\left(\mathrm{MH}, L^{\prime}\right)}-\lambda \ln \left(p^{\prime} / p_{\mathrm{a}}\right) \\
e_{\Gamma\left(\mathrm{MH}, L^{\prime}\right)}=e_{\Gamma}+\xi S_{\mathrm{MH}}^{\chi_{1}} L^{\prime \chi_{2}}
\end{gathered}
$$


In addition, the effects of $\mathrm{MH}$ saturation and phase state on the $\mathrm{MH}$ induced cohesion is suggested as:

$$
S=a S_{\mathrm{MH}}^{b} L^{\prime}
$$

where $a$ and $b$ are additional parameters controlling the effects of the new phase parameter and $\mathrm{MH}$ saturation on the $\mathrm{MH}$-induced cohesion.

\section{Calibration of the model}

The proposed model for MHBS contains 14 parameters, 8 of which describe the mechanical behaviors of the host sand and the remaining 6 account for the effects of $\mathrm{MH}$ saturation and the phase state. The value of the phase state parameter $L^{\prime}$ can be explicitly obtained by Eq. (11) for desired temperature and pore pressure. For instance, Table 1 shows the values of $L^{\prime}$ for desired temperature and pore pressure. Other parameters are determined in the following subsections.

Table 1: The values of $L^{\prime}$ for desired temperature and pore pressure

\begin{tabular}{ccc}
\hline Temperature $T[\mathrm{~K}]$ & Pore pressure $P[\mathrm{MPa}]$ & State parameter $L^{\prime}$ \\
\hline 274 & 10 & 0.0392 \\
274 & 15 & 0.0508 \\
278 & 5 & 0.0051 \\
278 & 10 & 0.0249 \\
283 & 10 & 0.0075 \\
\hline
\end{tabular}

\subsection{Calibration of parameters for the host sand}

The parameters $C_{i},(i=1,2,3,4)$ can be determined with a single triaxial compression test according to the procedure described by $\mathrm{Wu}$ and Bauer [27]. In this study, the material parameters determining $C_{i},(i=1,2,3$, 4) are $E_{\mathrm{i}} / \sigma_{\mathrm{c}}=180, \nu_{\mathrm{i}}=0.2, \psi_{\mathrm{crt}}=0^{\circ}$, and $\phi_{\mathrm{crt}}=31^{\circ}[7]$, where $E_{\mathrm{i}}$ is the initial tangent modulus; $\sigma_{\mathrm{c}}=1 \mathrm{MPa}$ is the confining pressure in the triaxial compression test; $\nu_{\mathrm{i}}$ is the initial Poisson ratio; $\psi_{\text {crt }}$ is the critical state dilatancy angle; and $\phi_{\text {crt }}$ is the critical state friction angle. The critical state parameters $e_{\Gamma}$ and $\lambda$ can be determined by fitting the critical state line of the host sand [7], as shown in Fig. 4. 
The parameters $\alpha$ and $\beta$ can be calibrated by fitting the peak strength and modulus of MHBS (in the same single triaxial compression test). The parameters for modeling of the host sand [7] are shown in Table 2.

Table 2: Model parameters for the host sand

\begin{tabular}{cc}
\hline Parameter & Value \\
\hline$C_{1}$ & -50 \\
$C_{2}$ & -53.46 \\
$C_{3}$ & -436.48 \\
$C_{4}$ & -147.73 \\
$e_{\Gamma}$ & 1.145 \\
$\lambda$ & 0.126 \\
$\alpha^{*}$ & 0.8 \\
$\beta^{*}$ & 3 \\
\hline
\end{tabular}

${ }^{*} \alpha$ and $\beta$ should be re-calibrated for MHBS.

\subsection{Calibration of parameters for $M H B S$}

Apart from the 8 model parameters for the host sand, 6 additional parameters are used to account for the effects of $\mathrm{MH}$ saturation, temperature, and pore pressure. As shown in Eqs. (15) and (16), both the MH saturation and the phase state influence the hydrate-induced cohesion and CSL on the $e-\ln p^{\prime}$ plane. For the MH-induced cohesion, the internal variable $S$ can be determined by back calculating the experimental data of the peak strength of MHBS [21]. As illustrated in Fig. 5, the internal variable $S$ is taken as the mean effective stress $p^{\prime}$ corresponding to zero deviatoric stress $q\left(q=\sigma_{1}^{\prime}-\sigma_{3}^{\prime}\right.$, with $\sigma_{1}^{\prime}$ and $\sigma_{3}^{\prime}$ being the maximum and minimum principal stresses, respectively) in the peak strength envelope. Therefore, the parameters $a$ and $b$ can be obtained by fitting the variation of $S$ against $S_{\mathrm{MH}}$ and $L^{\prime}$.

As illustrated in Fig. 6, the value of $e_{\Gamma\left(\mathrm{MH}, L^{\prime}\right)}$ in Eq. (15) is the intercept of the CSL on the $y$-axis. The parameters $\xi, \chi_{1}$, and $\chi_{2}$ can be obtained by fitting the variation of $e_{\Gamma\left(\mathrm{MH}, L^{\prime}\right)}$ against $S_{\mathrm{MH}}$ and $L^{\prime}$. Note that the parameters $\alpha$ and $\beta$ need to be re-calibrated for MHBS, which may be because the existence of $\mathrm{MH}$ alters the particle-scale property of sand grains. By fitting a single triaxial compression test on MHBS, the values of $\alpha$ and $\beta$ are determined as 0.3 and 5 , respectively. The remaining 5 parameters are presented in Table 3. 


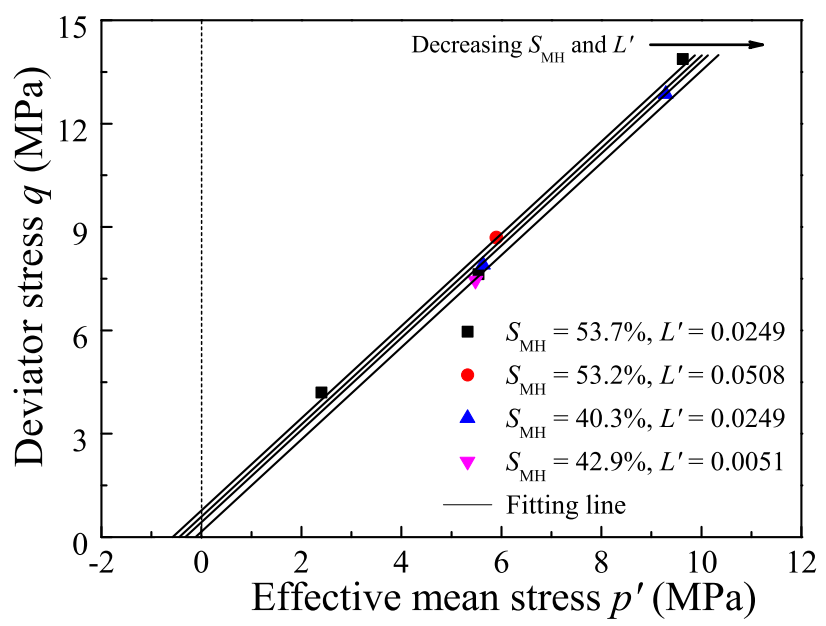

Figure 5: Peak strength envelopes of MHBS at different hydrate saturations (data from Hyodo et al. [7]).

Table 3: The remaining parameters for MHBS

\begin{tabular}{lccccc}
\hline Parameter & $a[\mathrm{MPa}]$ & $b$ & $\xi$ & $\chi_{1}$ & $\chi_{2}$ \\
\hline Value & 359 & 5.815 & 6.526 & 2.486 & 0.523 \\
\hline
\end{tabular}

\section{Model performance}

In this section, model predictions are compared with experimental results of MHBS. A series of numerical triaxial compression tests, considering different confining pressures, initial void ratios, $\mathrm{MH}$ saturations, temperatures, and pore pressures, are carried out to validate this model. The parameters used in the simulations are summarized in Tables 2 and 3.

\subsection{Model performance for the host sand}

Figures 7 and 8 show the comparisons between the predictions and experiments on host sand with different confining pressures and void ratios, respectively. In experiments, the strength and stiffness of MHBS increase with the increase of the effective confining pressure, while the dilatancy is weakened by the increase of the void ratio. In addition, a strain-softening behavior is observed under a lower effective confining pressure, i.e., $1 \mathrm{MPa}$, while strain-hardening behaviors are observed under higher effective confining pressures of 3 and $5 \mathrm{MPa}$. Overall, the model can well predict the 


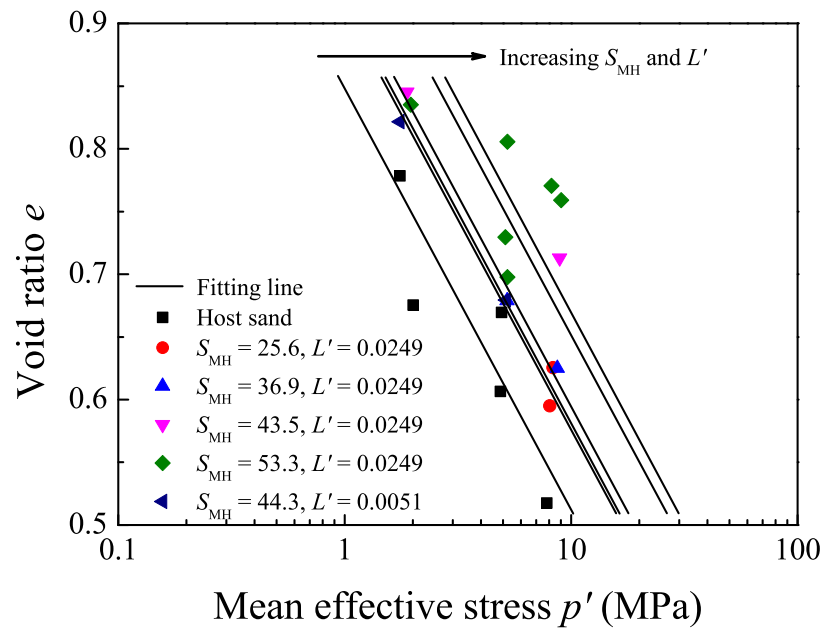

Figure 6: The effects of $S_{\mathrm{MH}}$ and $L^{\prime}$ on the critical state line (data from Hyodo et al.[7]).

stress-strain and volumetric responses of the host sand for different confining pressures and void ratios observed in experiments [7].

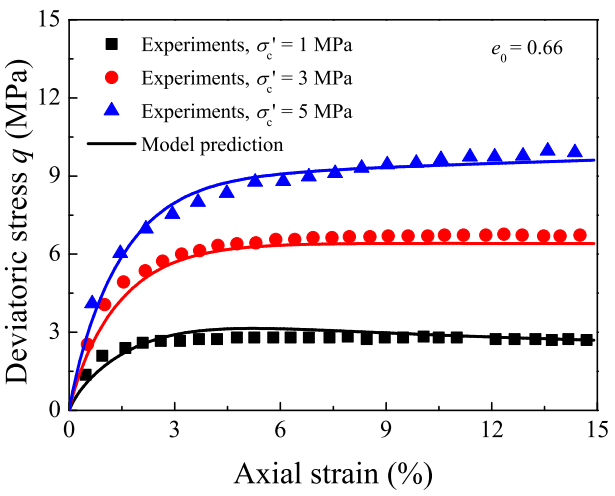

(a)

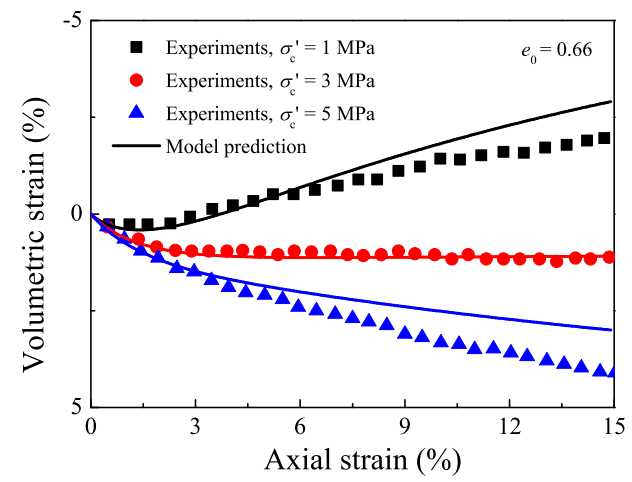

(b)

Figure 7: Comparisons between predicted and experimental results for the host sand with different effective confining pressures: (a) stress-strain and (b) volumetric responses (data from Hyodo et al. [7]).

\subsection{Model performance for $M H B S$}

Figure 9 presents the comparisons between experimental and the predicted results of MHBS with different $\mathrm{MH}$ saturations. Experimental results 


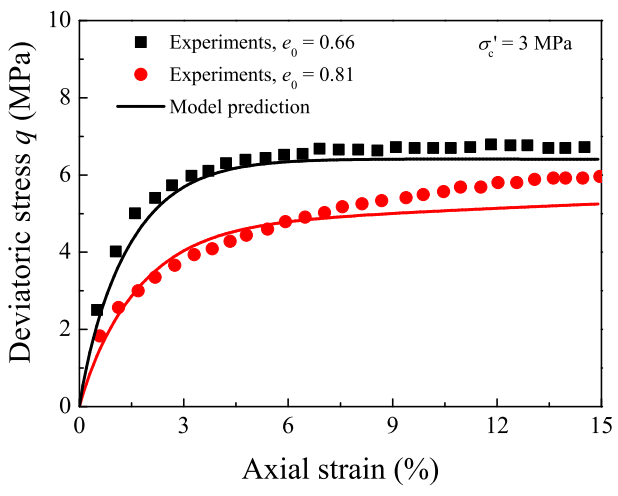

(a)

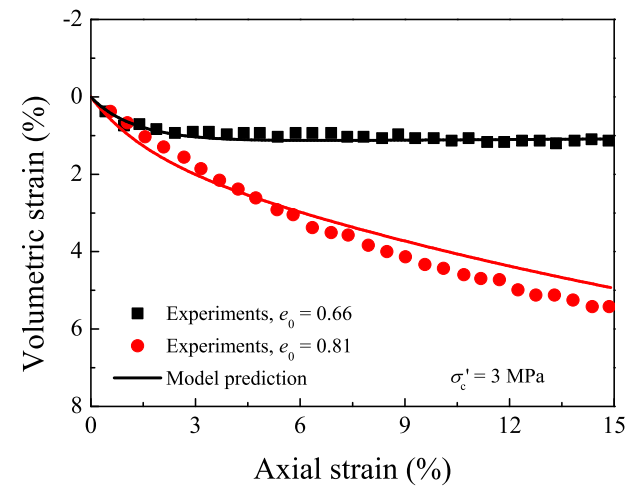

(b)

Figure 8: Comparisons between predicted and experimental results for the host sand with different void ratios: (a) stress-strain and (b) volumetric responses (data from Hyodo et al. [7]).

indicate that, with the increase of $S_{\mathrm{MH}}$, the MHBS samples show enhancement in strength, stiffness, and dilatancy. At an $\mathrm{MH}$ saturation of $53.1 \%$, a significant dilative behavior is observed regardless of the adopted high effective confining pressure $(5 \mathrm{MPa})$. Despite the slightly discrepancy, the model successfully captures the main effects of $S_{\mathrm{MH}}$ on the stress-strain behaviors and the volumetric responses of MHBS.

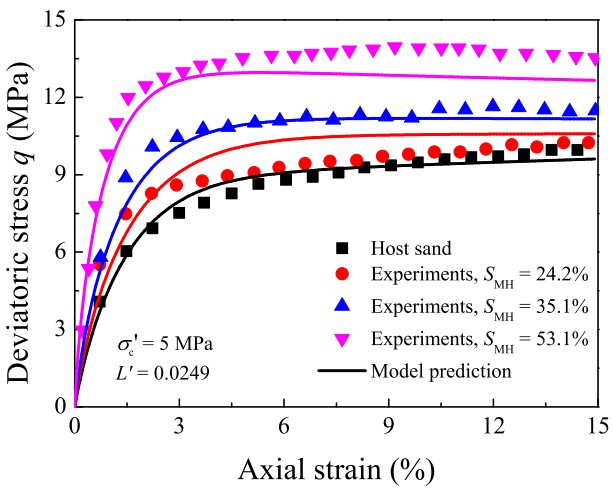

(a)

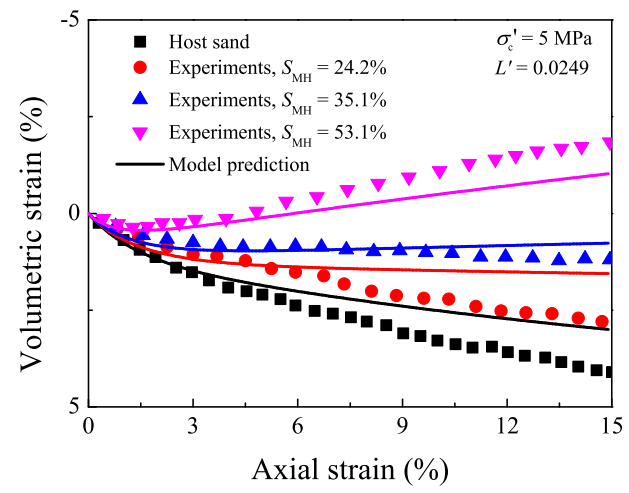

(b)

Figure 9: Comparisons between predicted and experimental results for MHBS with different MH saturations: (a) stress-strain and (b) volumetric responses (data from Hyodo et al. [7]).

Figures 10 and 11 illustrate the predicted mechanical behaviors of MHBS 
with different confining pressures and void ratios, respectively. It can be observed in experiments that for MHBS with $S_{\mathrm{MH}}$ of $53.7 \%$, the strength, stiffness, and dilatancy are enhanced by increasing effective confining pressures and decreasing void ratios. The proposed model can capture the changes of the stress-strain behaviors and volume change responses with varying effective confining pressures and void ratios. Admittedly, there are some discrepancies between the predicted stress-strain/volumetric responses and experimental data. The discrepancies maybe attributed to the effect of $\mathrm{MH}$ morphology, which is not considered in the current model. This effect will be discussed in Section 6.

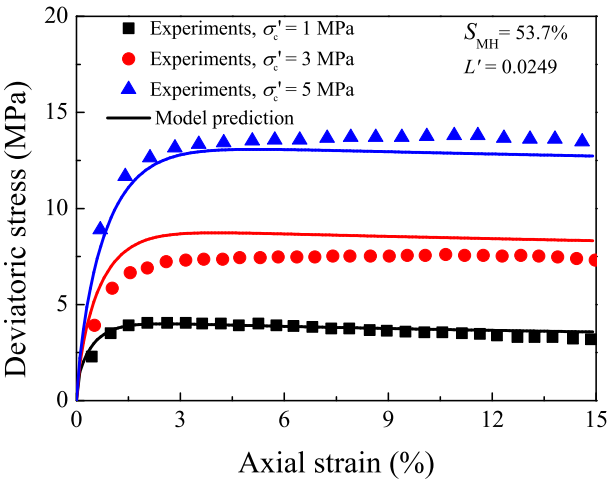

(a)

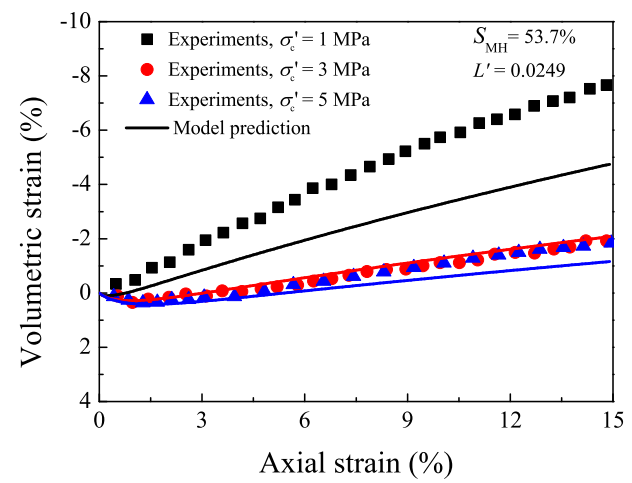

(b)

Figure 10: Comparisons between predicted and experimental results for MHBS with different confining pressures: (a) stress-strain and (b) volumetric responses (data from Hyodo et al. [7]).

Figures 12 and 13 compare the measured and predicted results of MHBS under different temperatures and pore pressures. Experimental results show that when pore pressure increase from $5 \mathrm{MPa}$ to $10 \mathrm{MPa}$, the stiffness of MHBS increase markedly; and the strength and dilatancy are also enhanced. In addition, when temperature decreases from $283 \mathrm{~K}$ to $274 \mathrm{~K}$, obvious increase in the stiffness, strength, and dilatancy of MHBS are observed. The comparisons of experimental and numerical results show that the proposed model is able to sufficiently capture the above effects. Overall, the results predicted by our model are close to the experimental data. 


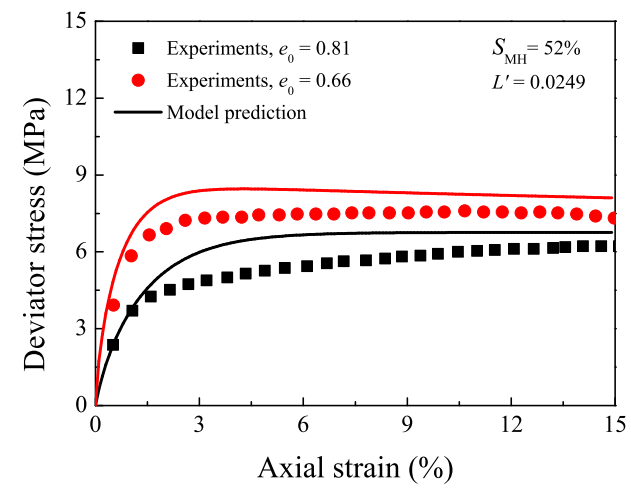

(a)

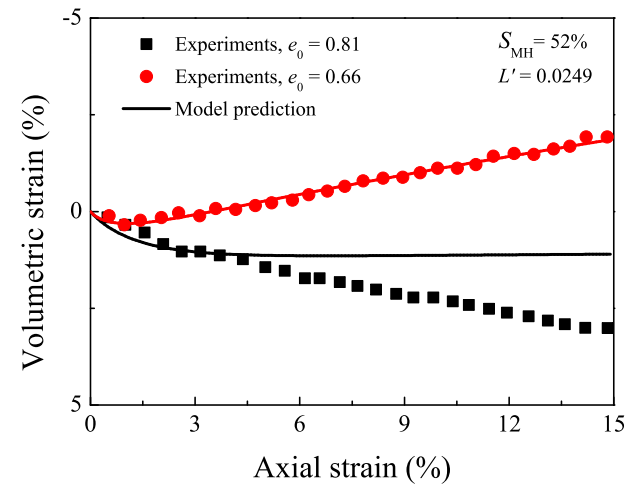

(b)

Figure 11: Comparisons between predicted and experimental results for MHBS with different void ratios: (a) stress-strain and (b) volumetric responses (data from Hyodo et al. [7]).

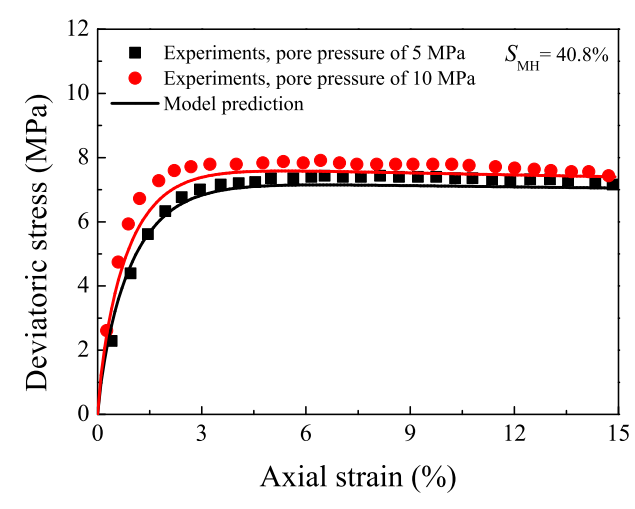

(a)

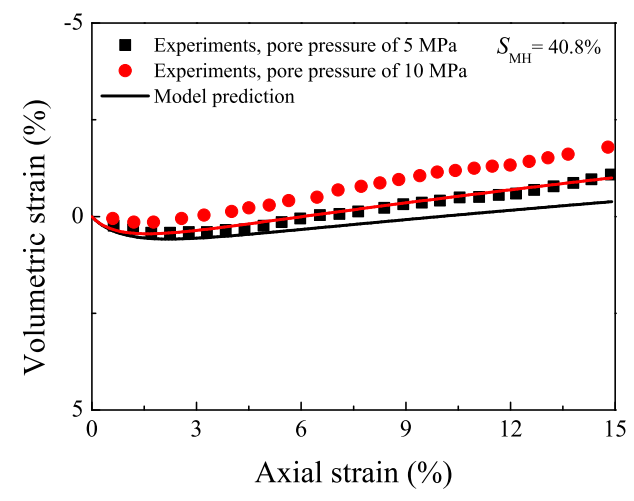

(b)

Figure 12: Comparisons between predicted and experimental results for MHBS with different pore pressures: (a) stress-strain and (b) volumetric responses (data from Hyodo et al. [7]).

\section{Discussion}

The mechanical behaviors of MHBS show a strong dependency on $\mathrm{MH}$ saturation. With the increase of $\mathrm{MH}$ saturation, a significant change in mechanical response will occur. Besides, the coupled effects of temperature and pore pressure are also important aspects of the mechanical behaviors of MHBS. So far, most of available constitutive models for MHBS take only the effect of MH saturation into account. One exception is the contribution made 


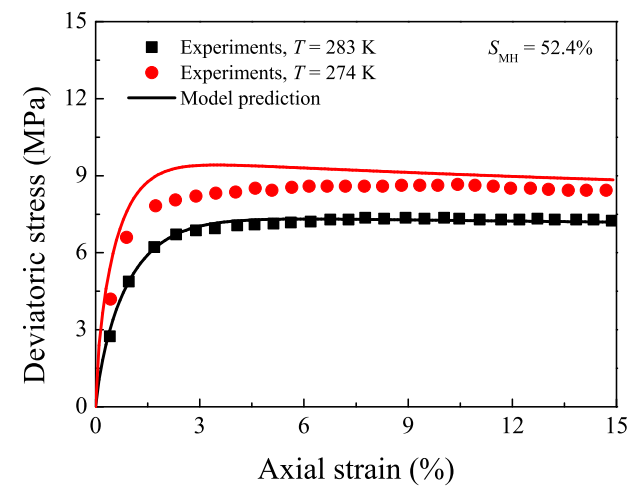

(a)

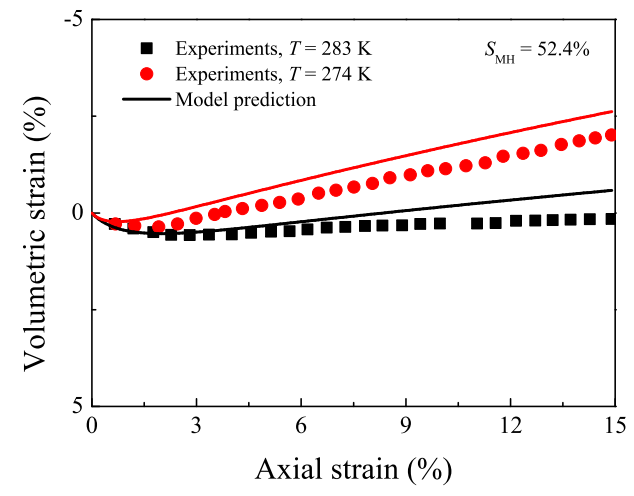

(b)

Figure 13: Comparisons between predicted and experimental results for MHBS with different temperatures: (a) stress-strain and (b) volumetric responses (data from Hyodo et al. [7]).

by Ng et al. [23], who consider the coupled effects of temperature and pore pressure by a phase parameter similar to that in this study. However, the phase parameter in [23] is determined in an indirect way (e.g., by iterative method), while the phase parameter proposed in this study can be determined straightforward by a simple function. In addition, the formulation of the proposed model is much more simple than that of most available constitutive models for MHBS. Therefore, the proposed hypoplastic model possess great advantages in modeling the salient mechanical behaviors of MHBS. Besides, the proposed phase parameter may provide convenience in future modeling of the coupled effects of temperature and pore pressure.

Apart from $\mathrm{MH}$ saturation, temperature, and pore pressure, the $\mathrm{MH}$ morphology is also an important factor in controlling the mechanical behaviors of MHBS. The MH morphology is usually categorized into four types: porefilling, load-bearing, cementation, and grain-coating [12]. In the past, the $\mathrm{MH}$ morphology is viewed to be controlled by hydrate formation environment. For example, in a free water environment, hydrate tends to form from dissolved gas molecules or gas bubbles, resulting in the pore-filling type or the load-bearing type; in a free gas environment, hydrate tends to form as the cementation type. Based on this idea, experiments performed in different hydrate formation environments (free gas/free water environment) $[7,8]$ were usually used for the validation of constitutive models in terms of different MH morphologies (cementation/pore-filling type) [23, 24, 51]. However, $\mathrm{MH}$ 
morphology cannot be identified simply by the hydrate formation environment. Based on high-quality tomography (e.g., micro-computed tomography and X-ray tomography), it is reported that pore-filling type $\mathrm{MH}$ is formed even in the free gas environment [52]. Moreover, reliable evidences that the transformation of $\mathrm{MH}$ morphology and coexistence of different $\mathrm{MH}$ morphologies are increasingly reported $[53,54]$. Therefore, cautions should be taken when using experimental data in terms of $\mathrm{MH}$ morphology since idealized pore-filling or cementation type MH may not physically exist.

The effect of MH morphology is not accounted for in this study because available experiments are not sufficient to isolate the effects of pore-filling, cementation, grain-coating, and load-bearing types of MH. Inevitably, the ignorance of $\mathrm{MH}$ morphology hinders the accuracy in model prediction. In addition, the accuracy in model prediction is also influenced by the adopted critical state parameter. Since the critical state parameters are determined at the end of shearing, where the critical state may not be reached [7], the performance of our model may be affected. To establish a comprehensive constitutive model for MHBS, the effect of MH morphology and the critical state behaviors of MHBS need further study.

\section{Conclusions}

A state-dependent critical state model is developed within the framework of hypoplasticity. The model is able to account for hydrate saturation, temperature, and pore pressure. A new phase parameter with explicit formulation is proposed, which includes the coupled effects of temperature and pore pressure on the mechanical behaviors of MHBS in a unified way. The model is validated with experimental results of MHBS obtained under a wide range of stress level, void ratios, hydrate saturation, temperature, and pore pressure. Comparisons of the predictions with experiments show the model is satisfactory in predicting the salient mechanical behaviors of MHBS.

Benefited from the non-linear tensorial functions, the hypoplastic model has the advantage of simplicity while retaining its ability to capture the salient behaviors of MHBS. These advantages make the hypoplastic model as an attractive alternative to the prevailing elastoplastic constitutive models for MHBS. 


\section{Acknowledgments}

The authors would like to acknowledge the National Natural Science Foundation of China with Grant Numbers 51639008 and 51890911, the H2020 Marie Skłodowska-Curie Actions RISE 2017 "HERCULES" with grant number 778360, and "FRAMED" with grant number 734485. The second author wishes to thank the Otto Pregl Foundation for financial support in Austria.

\section{Appendix}

The complete hypoplastic equations for MHBS are illustrated as follows:

$\stackrel{\circ}{\boldsymbol{\sigma}}=f_{\mathrm{s}}\left[C_{1}\left(\operatorname{tr} \boldsymbol{\sigma}_{\mathrm{T}}\right) \dot{\boldsymbol{\epsilon}}+\left(1-f_{\mathrm{d}}\right) C_{2}\left(\operatorname{tr} \boldsymbol{\sigma}_{\mathrm{T}}\right)(\operatorname{tr} \dot{\boldsymbol{\epsilon}}) \mathbf{I}+C_{3} \frac{\operatorname{tr}\left(\boldsymbol{\sigma}_{\mathrm{T}} \dot{\boldsymbol{\epsilon}}\right)}{\operatorname{tr} \boldsymbol{\sigma}_{\mathrm{T}}} \boldsymbol{\sigma}_{\mathrm{T}}+f_{\mathrm{d}} C_{4}\left(\boldsymbol{\sigma}_{\mathrm{T}}+\boldsymbol{\sigma}_{\mathrm{T}}^{*}\right)\|\dot{\boldsymbol{\epsilon}}\|\right]$ where

$$
\left\{\begin{array}{l}
\boldsymbol{\sigma}_{\mathrm{T}}=\boldsymbol{\sigma}+S \mathbf{I} \\
S=a S_{\mathrm{MH}}^{b} L^{\prime} \\
f_{\mathrm{s}}=\frac{\exp \left[\beta\left(e_{\mathrm{crt}}-e\right)\right]}{(1+r)^{2}} \\
f_{\mathrm{d}}=\left(\frac{e}{e_{\mathrm{crt}}}\right)^{\alpha} \\
e_{\mathrm{crt}}=e_{\Gamma}\left(\mathrm{MH}, L^{\prime}\right)-\lambda \ln \left(p^{\prime} / p_{\mathrm{a}}\right) \\
e_{\Gamma\left(\mathrm{MH}, L^{\prime}\right)}=e_{\Gamma}+\xi S_{\mathrm{MH}}^{\chi_{1}} L^{\prime \chi_{2}}
\end{array}\right.
$$

In the above equations, $\boldsymbol{\sigma}_{\mathrm{T}}$ is the transformed stress tensor, $\boldsymbol{\sigma}$ is the Jaumann stress rate, $\boldsymbol{\sigma}$ is the Cauchy stress tensor, $\dot{\boldsymbol{\epsilon}}$ is the stretching tensor, I is the second-order unit tensor, $S$ is the internal variable $e$ is the current void ratio, $e_{\text {crt }}$ is the critical state void ratio, $r$ is the stress ratio $\left\|\boldsymbol{\sigma}^{*}\right\| /(\operatorname{tr} \boldsymbol{\sigma}), L^{\prime}$ is the phase state parameter, $e_{\Gamma\left(\mathrm{MH}, L^{\prime}\right)}$ is the critical state void of MHBS at the atmospheric pressure, $e_{\Gamma}$ is the critical state void of sand at the atmospheric pressure $p_{\mathrm{a}}$, and $p^{\prime}$ is the effective mean stress. The parameters of the model are $C_{1}, C_{2}, C_{3}, C_{4}, e_{\Gamma}, \lambda, \alpha, \beta, a, b, \xi, \chi_{1}, \chi_{2}$, and $L^{\prime}$.

\section{References}

[1] Waite WF, Santamarina JS, Cortes DD, Dugan B, Espinoza DN, Germaine J, Jang J, Jung JW, Kneafsey TJ, Shin H, Soga K, Winters WJ, Yun TS. Physical properties of hydrate-bearing sediments. Rev Geophys 2009; 47(4). 
[2] Soga K, Lee SL, Ng MYA, Klar A. Characterisation and engineering properties of methane hydrate soils. Characterisation and engineering properties of natural soils 2006; 4:2591-1642.

[3] Boswell R. Is gas hydrate energy within reach? Science 2009; 325(5943):957-958.

[4] Zhang XH, Lu XB, Shi YH, Xia Z, Liu WT. Centrifuge experimental study on instability of seabed stratum caused by gas hydrate dissociation. Ocean Eng 2015; 105:1-9.

[5] Rutqvist J, Moridis GJ, Grover T, Collett T. Geomechanical response of permafrost-associated hydrate deposits to depressurization-induced gas production. J Petrol Sci Eng 2015; 67(1-2):1-12.

[6] Nixon MF, Grozic JLH. Submarine slope failure due to gas hydrate dissociation: a preliminary quantification. Can Geotech J 2007; 44(3):314325 .

[7] Hyodo M, Yoneda J, Yoshimoto N, Nakata Y. Mechanical and dissociation properties of methane hydrate-bearing sand in deep seabed. Soils Found 2013; 53(2):299-314.

[8] Masui A, Haneda H, Ogata Y, Aoki K. Effects of methane hydrate formation on shear strength of synthetic methane hydrate sediments. 2005; In The Fifteenth International Offshore and Polar Engineering Conference. International Society of Offshore and Polar Engineers.

[9] Choi JH, Dai S, Lin JS, Seol Y. Multistage triaxial tests on laboratoryformed methane hydrate-bearing sediments. J Geophys Res Solid Earth 2018; 123(5):3347-3357.

[10] Madhusudhan BN, Clayton CRI, Priest JA. The effects of hydrate on the strength and stiffness of some sands. J Geophys Res Solid Earth $2019 ; 124(1): 65-75$.

[11] Dong L, Li YL, Liao HL, Liu CL, Chen Q, Hu GW, Liu LL, Meng QG. Strength estimation for hydrate-bearing sediments based on triaxial shearing tests. J Pet Sci Eng 2019; 106478. 
[12] Yun TS, Francisca FM, Santamarina JC, Ruppel C. Compressional and shear wave velocities in uncemented sediment containing gas hydrate. Geophys Res Lett 2005; 32(10).

[13] Hyodo M, Li YH, Yoneda J, Nakata Y, Yoshimoto N, Nishimura A, Song YC. Mechanical behavior of gas-saturated methane hydrate-bearing sediments. J Geophys Res Solid Earth 2013; 118(10):5185-5194.

[14] Yu F, Song Y, Liu W, Li Y, Lam W. Analyses of stress strain behavior and constitutive model of artificial methane hydrate. J Petrol Sci Eng 2011; 77(2):183-188.

[15] Miyazaki K, Tenma N, Aoki K, Yamaguchi T. A nonlinear elastic model for triaxial compressive properties of artificial methane-hydrate-bearing sediment samples. Energies 2012; 5(10):4057-4075.

[16] Song YC, Zhu YM, Liu WG, Li YH, Lu Y, Shen ZT. The effects of methane hydrate dissociation at different temperatures on the stability of porous sediments. J Petrol Sci Eng 2016; 147:77-86.

[17] Klar A, Soga K, Ng MYA. Coupled deformation-flow analysis for methane hydrate extraction. Géotechnique 2010; 60(10):765-776.

[18] Pinkert S, Grozic JLH. Prediction of the mechanical response of hydratebearing sands. J Geophys Res: Solid Earth 2014; 119(6):4695-4707.

[19] Pinkert S, Grozic JLH, Priest JA. Strain-softening model for hydratebearing sands. Int J Geomech 2015; 15(6):04015007.

[20] Pinkert S. Rowes stress-dilatancy theory for hydrate-bearing sand. Int $J$ Geomech 2016; 17(1):06016008.

[21] Shen J, Chiu CF, Ng CWW, Lei GH, Xu J. A state-dependent critical state model for methane hydrate-bearing sand. Comput Geotech 2016; 75:1-11.

[22] Uchida S, Xie XG, Leung YF. Role of critical state framework in understanding geomechanical behavior of methane hydrate-bearing sediments. J Geophys Res: Solid Earth 2016; 121(8):5580-5595. 
[23] Ng CWW, Baghbanrezvan S, Kadlicek T, Zhou C. A state-dependent constitutive model for methane hydrate-bearing sediments inside the stability region. Géotechnique 2019; 39(9):1-15.

[24] Sánchez M, Gai XR, and Santamarina JC. A constitutive mechanical model for gas hydrate bearing sediments incorporating inelastic mechanisms. Comput Geotech 2017; 84:28-46.

[25] Lin JS, Seol Y, Choi JH. An SMP critical state model for methane hydrate-bearing sands. Int J Numer Anal Methods Geomech 2015; 39(9):969-987.

[26] Kolymbas D. An outline of hypoplasticity. Arch Appl Mech 1991; 61(3):143-151.

[27] $\mathrm{Wu} \mathrm{W,} \mathrm{Bauer} \mathrm{E.} \mathrm{A} \mathrm{simple} \mathrm{hypoplastic} \mathrm{constitutive} \mathrm{model} \mathrm{for} \mathrm{sand.} \mathrm{Int}$ J Numer Anal Methods Geomech 1994; 18(12):833-862.

[28] Wu W, Lin J, Wang XT. A basic hypoplastic constitutive model for sand. Acta Geotech 2017; 12(6):1373-1382.

[29] Wang S, Wu W, Yin ZY, Peng C, He XZ. Modelling the time-dependent behaviour of granular material with hypoplasticity. Int J Numer Anal Methods Geomech 2018; 42(12):1331-1345.

[30] Tang Y, Wu W, Yin KL, Wang S, Lei GP. A hydro-mechanical coupled analysis of rainfall induced landslide using a hypoplastic constitutive model. Comput Geotech 2019; 112:284-292, .

[31] Mašín D. A hypoplastic constitutive model for clays. Int J Numer Anal Methods Geomech 2005; 29(4):311-336.

[32] Huang WX, Wu W, Sun DA, Sloan S. A simple hypoplastic model for normally consolidated clay. Acta Geotech 2006; 1(1):15-27.

[33] Xu GF, Wu W, Qi JL. An extended hypoplastic constitutive model for frozen sand. Soils Found 2016; 56(4):704-711.

[34] Guo XG, Peng C, Wu W, Wang YQ. A hypoplastic constitutive model for debris materials. Acta Geotech 2016; 11(6):1217-1229. 
[35] Zhang XH, Lin J, Lu XB, Liu LL, Liu CL, Li MY, Su YW. A hypoplastic model for gas hydrate-bearing sandy sediments. Int J Numer Anal Methods Geomech 2018; 42(7):931-942.

[36] Wu W, Kolymbas D. Hypoplasticity then and now. 2000; In Constitutive modelling of granular materials, pages 57-105. Springer.

[37] Wu W, Bauer E, Kolymbas D. Hypoplastic constitutive model with critical state for granular materials. Mech Mater 1996; 23(1):45-69.

[38] Wang S, Wu W, Peng C, He XZ, Cui DS. Numerical integration and fe implementation of a hypoplastic constitutive model. Acta Geotech 2018; 13(6):1265-1281.

[39] Hyodo M, Nakata Y, Yoshimoto N, Fukunaga M, Kubo K, Nanjo Y, Matsuo T, Hyde AFL, Nakamura K. Triaxial compressive strength of methane hydrate. 2002; In The Twelfth International Offshore and Polar Engineering Conference. International Society of Offshore and Polar Engineers.

[40] Jiang MJ, Zhu FY, Liu F, Utili S. A bond contact model for methane hydrate-bearing sediments with interparticle cementation. Int $J$ Numer Anal Methods Geomech 2014; 38(17):1823-1854.

[41] Shen ZF, Jiang MJ. DEM simulation of bonded granular material. part ii: extension to grain-coating type methane hydrate bearing sand. Comput Geotech, 75:225-243, 2016.

[42] Jiang MJ, Shen ZF, Wu D. CFD-DEM simulation of submarine landslide triggered by seismic loading in methane hydrate rich zone. Landslides 2018; 15(11):2227-2241.

[43] Jiang MJ, Liu J, Shen ZF. DEM simulation of grain-coating type methane hydrate bearing sediments along various stress paths. Eng Geol 2019; 105280.

[44] Duan ZH, Li D, Chen YL, Sun R. The influence of temperature, pressure, salinity and capillary force on the formation of methane hydrate. Geosci Front 2011; 2(2):125-135. 
[45] Makogon TY, Sloan EDJ. Phase equilibrium for methane hydrate from 190 to 262 K. J Chem Eng Data 1994; 39(2):351-353.

[46] Jager MD, Sloan ED. The effect of pressure on methane hydration in pure water and sodium chloride solutions. Fluid Phase Equilib 2001; 185(1-2):89-99.

[47] Maekawa T. Equilibrium conditions for gas hydrates of methane and ethane mixtures in pure water and sodium chloride solution. Geochem $J$ 2001; 35(1):59-66.

[48] Sun SC, Ye YG, Liu CL, Xiang FK, Ma Y. Pt stability conditions of methane hydrate in sediment from south china sea. J Nat Gas Chem 2011; 20(5):531-536.

[49] Sabil KM, Nashed O, Lal B, Ismail L, Japper-Jaafar A. Experimental investigation on the dissociation conditions of methane hydrate in the presence of imidazolium-based ionic liquids. J Chem Thermodyn 2015; 84:7-13.

[50] Cha MJ, Hu Y, Sum AK. Methane hydrate phase equilibria for systems containing $\mathrm{NaCl}, \mathrm{KCl}$, and $\mathrm{NH} 4 \mathrm{Cl}$. Fluid Phase Equilib 2016; 413:2-9.

[51] Yan RT, Wei CF. Constitutive model for gas hydrate-bearing soils considering hydrate occurrence habits. Int J Geomech 2017; 17(8):04017032.

[52] Chaouachi M, Falenty A, Sell K, Enzmann F, Kersten M, Haberthür D, Kuhs WF. Microstructural evolution of gas hydrates in sedimentary matrices observed with synchrotron X-ray computed tomographic microscopy. Geochem Geophys Geosyst 2015; 16(6):1711-1722.

[53] Sell K, Saenger EH, Falenty A, Chaouachi M, Haberthür D, Enzmann F, Kuhs WF, Kersten M. On the path to the digital rock physics of gas hydrate-bearing sediments-processing of in situ synchrotrontomography data. Solid Earth 2016; 7(4):1243-1258.

[54] Lei L, Seol Y, Choi JH, Kneafsey TJ. Pore habit of methane hydrate and its evolution in sediment matrix-laboratory visualization with phasecontrast micro-CT. Mar Pet Geol 2019; 104:451-467. 